\title{
Coronary Endarterectomy Improve Left Ventricle Ejection Fraction After Coronary Artery Bypass Grafting Surgery
}

\author{
Manochihr Timorian, Mirwais Amiri, Abdullah Alimi \\ Department of Cardiothoracic and Vascular Surgery and Adult Cardiology Amiri Medical Complex, Kabul, Afghanistan \\ Email address: \\ manochihr@yahoo.com (M. Timorian)

\section{To cite this article:} \\ Manochihr Timorian, Mirwais Amiri, Abdullah Alimi. Coronary Endarterectomy Improve Left Ventricle Ejection Fraction After Coronary \\ Artery Bypass Grafting SURGERY. International Journal of Cardiovascular and Thoracic Surgery. Vol. 5, No. 2, 2019, pp. 41-46. \\ doi: $10.11648 /$ j.ijcts.20190502.13
}

Received: March 28, 2019; Accepted: May 28, 2019; Published: June 10, 2019

\begin{abstract}
The diffusely diseased coronary artery is a challenge for cardiac surgeons, although coronary endarterectomy is an option for surgical reconstruction of a diffusely diseased vessel. It may assures complete revascularization of myocardium in case of diffusely diseased vessels and prevent residual ischemia but it has not been widely used. recently cardiac surgeons are performing and increasing number of coronary artery endarterectomy and it has evolved as an important adjuvant procedure in coronary artery bypass grafting surgery. we reviewed the early clinical and hemodynamic out comes with echocardiography of 22 patients undergoing coronary artery endarterectomy of left anterior descending artery (LAD) diagonal branches (D1 or D2) and right coronary artery and posterior descending artery (RCA and PDA) with patch plasty method using left internal mammary artery (LIMA) and saphenous vein graft (SVG) between January 2017 and June 2018. mean follow up of all patients were 3-6 months postoperatively, Left internal mammary artery (LIMA) anastomosed to left anterior descending artery (LAD) with endarterectomy and artery patch plasty in $12(54,5 \%)$ patients, saphenous vein graft (SVG) anastomosed to left anterior descending artery (LAD) with endarterectomy and vein patch plasty in 2 (9\%) patients, and saphenous vein graft (SVG) to Right coronary artery (RCA) Diagonal (D1) and posterior descending artery (PDA) with endarterectomy and vein patch plasty in $9(40.9 \%)$ patients. Postoperative mortality was $0 \%$ and echocardiographic assessment done by modified Simpson method on outpatient department (OPD) basis, 7 patients had left ventricle ejection fraction of (50-55\%) preoperatively with no changes on postoperative period and 7 patients had improvement of left ventricle ejection fraction from (30-35\%) preoperatively to (40-45\%) in postoperative period. principle goal in coronary artery bypass grafting surgery (CABG) is to achieve complete revascularization of diseased coronary arteries, in particular the left anterior descending artery (LAD) is very important vessel because incomplete revascularization of the LAD has been proven to be a predictor of worse mortality after coronary artery bypass grafting surgery (CABG). Recent publications have revealed that coronary endarterectomy is a safe procedure and demonstrated favorable long term out comes. In our experience coronary endarterectomy has been performed with good clinical and hemodynamic out comes with echocardiography.
\end{abstract}

Keywords: Cardiac Surgery, Coronary Artery Bypass Grafting Surgery, Coronary Endarterectomy

\section{Introduction}

Diffuse coronary artery atherosclerosis can be defined as "consecutive or longitudinal" and "complete or partial" obstruction in coronary vessels. Most of the patients with diabetes, hyperlipidemia, chronic renal insufficiency, connective tissue disease, and multistented coronary arteries have diffuse atherosclerotic lesions in the coronary territory. Viable large myocardium without necrosis is the only coronary bypass indication in these patients, because it is very difficult to find any healthy area for anastomosis. This type of coronary occlusion frequently stimulates the formation of collateral vessels that protect against extensive myocardial ischemia. The choice of a surgical method also depends on the nature of the coronary artery, and multisegment plaques and healthy-area intervals simplify complete revascularization. On the other hand, a more aggressive treatment modality should be preferred when no soft site can be identified for arteriotomy or there is an extensively diseased area that is not amenable to grafting. 
The less invasive techniques are "don't touch the plaque" techniques (jumping multi-bypass, sequential bypass, hybrid interventions). Sometimes an aggressive diffuse plaque formation needs to be treated with "touch the plaque" techniques (long-segment anastomosis, patch-plasty, endarterectomy \pm patch-plasty). In simple forms, a limited long-segment anastomosis of conduits eliminates the occlusion of the limited atherosclerotic plaque where the whole lesion is opened and cross-covered by the graft. In the accelerated form of coronary arteriosclerosis, the atherosclerotic plaque appears widespread and the full-length lumen of the coronary artery can get very narrow or occluded totally. The long-segment lesion is usually calcified and it inhibits any kind of stitching; however, the plaque can be separated easily from the arterial wall in order to create an appropriate lumen in the total occluded coronary artery. Because the aggressive endarterectomy increases the operation risk, the arteriotomy should be extended until the normal lumen with normal intima in the distal segment of the coronary artery. In general, severity and distribution of coronary arteriosclerosis tend to increase with time but the rate of increase is highly variable and difficult to predict. Although diffuse atherosclerosis is severe enough, it is uncommon to render any patient unsuitable for surgery.

\section{Etiology}

Most of the patients with diabetes, hyperlipidemia, chronic renal insufficiency, connective tissue disease and multistented coronary arteries have diffuse atherosclerotic lesions in the coronary territory. All of these diseases affect and accelerate coronary arteriosclerosis differently. Restenosis after first coronary artery bypass grafting surgery (CABG) can also be a reason for the diffuse coronary atherosclerosis, but usually these patients have ungraftable diffuse diseased coronary vasculature and none of the specific revascularization methods can be used.

\subsection{Diabetes Mellitus}

Diabetes mellitus increases the incidence of coronary artery disease two to four times as much and accelerates the nature of the atherosclerosis. The nature of coronary artery disease in diabetic patients is clinically challenging because it causes an extensive and diffuse multivessel involvement. Hyperglycemia is directly related to the atherosclerotic development, progression, and instability due to induced endothelial dysfunction (abnormal nitric oxide biology, increased endothelin and angiotensin II, reduced prostacyclin activity), abnormalities in lipid metabolism (high triglyceride and LDL-cholesterol, low HDLcholesterol), systemic inflammation (increased oxidative stress, accumulation of advanced glycation and products), and disorders in the proteo-fibrinolytic system and platelet biology (thrombosis). Hyperglycemia can deplete the cellular NADPH pool and induce with high levels of fatty acids to oxidative stress on phospholipids and proteins. Insulin resistance is the main actor to the endothelial dysfunction in type II diabetes, and endothelial dysfunction is closely complicated with microangiopathy and atherosclerosis in diabetic patients. Endothelial dysfunction decreases the capacity of nitric oxide synthase enzyme and depleted nitric oxide, which effects endothelial celldependent vasodilatation. Overexpression of growth factors causes endothelial cells and vascular smooth muscle proliferation. All of these negative changes accelerate atherosclerosis in all arterial territories, and the involvement of coronary arteries can be very extensive and diffuse with either serious jumping stenoses or longsegment narrowing with/without occlusion. The optimal strategy of coronary revascularization is controversial, but CABG has better long-term survival and freedom from reinterventions. Diabetic patients have a higher restenosis rate after stent implantation and also progression of diffuse disease after stent implantation forms new lesions in diabetic patients than non-diabetic patients more often. Clinical outcomes in CABG patients are similar for diabetic and nondiabetic patients, while outcomes after stent could be worse for diabetic patients. In diabetic patients with multivessel coronary artery disease, rates of death and myocardial infarction in 5 years are significantly lower in patients treated with $\mathrm{CABG}$ due to more complete revascularization, which bypasses several lesions and prevents coronary territory against progressive proximal coronary stenosis. On the other hand, the operative risk in patients with diabetes might be a consequence of a preoperatively endothelial dysfunction and an inflammatory response to extracorporeal circulation characterized by an impaired release of interleukin- 6 and increased turnover of E-selectin. Simple distal anastomosis for each coronary artery cannot be enough to supply blood along the coronary territory, and most of the diabetic patients with diffuse multivessel coronary artery disease require specific surgical revascularization modalities, which can increase perioperative myocardial damage and operative mortality.

\subsection{Hypercholesterolemia}

Cholesterol is one of the most important risk factors for the development of premature coronary artery disease, which is characterized without any serious intravascular stenosis. Cholesterol levels and coronary artery disease show a strong and linear relationship, whereas cholesterol levels even in the normal range may inhibit endothelium-dependent vasodilatation in all arterial beds. The pathogenesis of atherosclerosis in the obese population can be related to metabolic syndrome associated with insulin intolerance and dyslipidemia, which cause endothelial dysfunction with decreasing nitric oxide production. Lowering of LDLcholesterol rather than moderate weight loss is more effective to improve endothelial function, because the coronary vasculature is affected by the atherosclerosis process, and the most atherosclerotic lesions are associated with remarkable neovascularization of the vasa vasorum, which can cause intra-plaque rupture and bleeding. Hypercholesterolemia is one of the most important factors to stimulate this process 
and its role begins in the early atherosclerotic remodeling before plaque formation. Hyperlipidemia-related coronary lesions are very predisposed to spread lengthways coronary territory and cause diffuse stenosis or occlusion, and calcification is usually associated with this type of atherosclerosis.

\subsection{End-stage Renal Disease}

A strong relationship subsists between chronic renal failure and coronary artery disease, and atherosclerosis can be accelerated in patients with end-stage renal disease due to multifactorial reasons Increased oxidative stress, hyperhomocysteinemia, hyperlipidemia, hyperglycemia and others are also important comorbidities. The main pathology is the impairment of endothelium-dependent vasodilatation. Dialysis-dependent renal failure patients undergoing coronary artery bypass grafting surgery (CABG) can have a greater degree of distal and/or diffuse coronary artery disease burden compared with matched patients with silent renal failure. The diffuseness of coronary atherosclerosis in patients with end-stage renal disease can be severe and the intraluminal lesions are usually calcified. Extensive calcification of all arterial structures in the body can inhibit conventional coronary artery bypass grafting surgery (CABG) strategies, which increase surgical outcomes. Impaired distal runoff of the coronary arteries is another strong independent predictor of operative mortality. All kinds of complex anastomotic techniques can be used in these patients, and endarterectomy can be very easy to perform to get adequate distal run-off. Restenosis after coronary artery bypass grafting surgery (CABG) is not uncommon in this group of patients, especially if saphenous vein is used.

\subsection{Connective Tissue Disease}

Several connective tissue diseases (systemic lupus erythematosus, rheumatoid arthritis, systemic sclerosis, Takayasu disease) are characterized by vascular dysfunction and excessive fibrosis. The presence of coronary microvascular dysfunction is the common pathologic change in various chronic inflammatory diseases. Cardiac manifestation of these chronic diseases can be estimated lower, because most of them are asymptomatic. Diffuse form of these pathologies has a distressed clinical course with severe organ involvement. First, an endothelial injury occurs early in the disease process leading to endothelial dysfunction. Myofibroblasts drawn into the arterial wall by cellular growth factors contribute to the thickening of the intimal layer, compromising regional blood flow by narrowing the arterial lumen. In the absence of epicardial coronary stenosis, the abnormal coronary flow is dependent on the structural remodeling of the small coronary arteries and arterioles. Aggressive surgical interventions are usually ineffective, but multi-anastomoses can be applicable. Because diffuse atherosclerosis shows strict adhesions between arterial wall layers, endarterectomy can never satisfy to load out the intra-arterial lumen for appropriate anastomosis

\subsection{Multistented Coronary Arteries}

The problem of restenosis after stenting represents a special case of arterial hyperplastic disease and the in-stent restenosis is made from myxomatous tissue, whereas accelerated intimal hyperplasia occludes the distal segment of the same coronary vessel after stenting. Availability of access to healthy coronary wall for revascularization is usually feasible in patients receiving a single stent implantation in one or each coronary artery. However, the distal vascular bed of multi-stented coronary artery is often influenced by the accelerated atherosclerosis and diffusely diseased where it is impossible to find any healthy area for distal anastomosis. Sometimes, open endarterectomy with removal of stent (s) can remain the last option for surgical revascularization.

Surgical treatment techniques: Diffuse atherosclerosis has been highly widespread among patients with coronary artery disease in the last two decades, because simple lesions are usually treated with stent interventions in the early phase of the coronary pathology. Diffuse coronary lesion and reduced coronary flow reserve can be silent due to several collaterals, but it might result in severe functional limitation, chronic low-level ischemia, and myocardial remodeling. Low-level ischemia can be a potential driver of both first coronary vasomotor and myocardial dysfunction, and then remodeling in heart failure with preserved ejection fraction [14]. Diffuse atherosclerosis and microvascular dysfunction-associated coronary artery disease comorbid conditions may guide new, more effective, aggressive, and therapeutic interventions for global cardiovascular risk reduction due to complete revascularization. There is no difference in event-free survival between coronary artery bypass grafting surgery (CABG) or stent implantation in patients with high coronary flow reserve; however, coronary artery bypass grafting surgery $(\mathrm{CABG})$ is significantly more effective than stent in patients with low coronary flow reserve. Diffuseness of coronary artery disease is a serious risk factor for early and late adverse events after coronary revascularization, but the acceptable strategy should be complete revascularization. Standard bypass method (finding an appropriate lumen and performing anastomosis) is usually not possible in the diffusely diseased coronary arteries, and such a region, which may be found at most distal, cannot be expected to bring any benefit. For this reason, in such cases, it is required to apply a complex method other than standard bypass method. [7] When the atherosclerotic stenosis is local, it is technically possible and easy to revascularize the distal segment directly, but in diffuse coronary artery disease or in the presence of diffuse stenotic regions, different techniques should be implemented for complete revascularization.

A. No-touch the plaque techniques.

1. Jumping bypass (the same coronary artery).

a) With multiple grafts.

b) With a single graft.

c) With a composite graft. 
d) With a bifurcated graft.

2. Sequential bypass (multiple coronary arteries).

a) With a single graft.

b) With a composite graft.

c) With a bifurcated graft.

3. Hybrid revascularization (different coronary arteries).

B. Touch the plaque techniques.

1. Long-segment anastomosis.

2. Patch-plasty.

3. Endarterectomy \pm patch-plasty.

a) Closed.

b) Open.

\section{Patients and Method.}

\subsection{Patients}

between January 2017 and June 2018, 22 consecutive patients with diffuse coronary artery diseases diabetes mellitus, hypertension and dyslipidemia underwent coronary artery bypass grafting surgery with coronary endarterectomy and patch plasty at department of cardiothoracic and vascular surgery amiri medical complex Kabul Afghanistan as a single center study.

There were $19(86.36 \%)$ male patients and $3(13.63 \%)$ female patients, age was between (38-75) years. there were $10(45.4 \%)$ patient with uncontrolled diabetes mellitus, 6 $(27.2 \%)$ with chronic hypertension and all of them $(100 \%)$ present with dyslipidemia, renal function test were deranged in $5(22.7 \%)$ patients (creatinine $>2.2)$, left ventricle ejection fraction were (30-55\%) preoperatively.

Indication for coronary artery bypass grafting surgery made by coronary angiography in all patients.

\subsection{Operative Procedure}

The operation performed by on pump coronary artery bypass grafting surgery with aortic and two stage single right atrial cannulation with moderate hypothermia (28-30) and heart arrested with antigrade and retrograde cold cardioplegia, endarterectomy done by touch the plaque technique (open and closed). left internal mammary artery (LIMA) anastmosed to left anterior descending artery (LAD) with endarterectomy and artery patch plasty in 12 (54, 5\%) patients, saphenous vein graft (SVG) anastomosed to left anterior descending artery (LAD) with endarterectomy and vein patch plasty in $2(9 \%)$ patients and saphenous vein graft (SVG) to Right coronary artery (RCA) Diagonal (D1) and posterior descending artery (PDA) with endarterectomy and vein patch plasty in $9(40.9 \%)$ patients. All patients received high dose of antiplatelet and anticoagulant 6 hours postoperatively.

\subsection{Postoperative Complication}

No significant postoperative complication was present, only two patients were re explored due to postoperative bleeding. Intra-aortic balloon pump used for 3 patients because of low ejection fraction. Intensive care unit stay was
3 to 6 days and overall hospital stay was 7 to 12 days, there was no postoperative mortality at all.

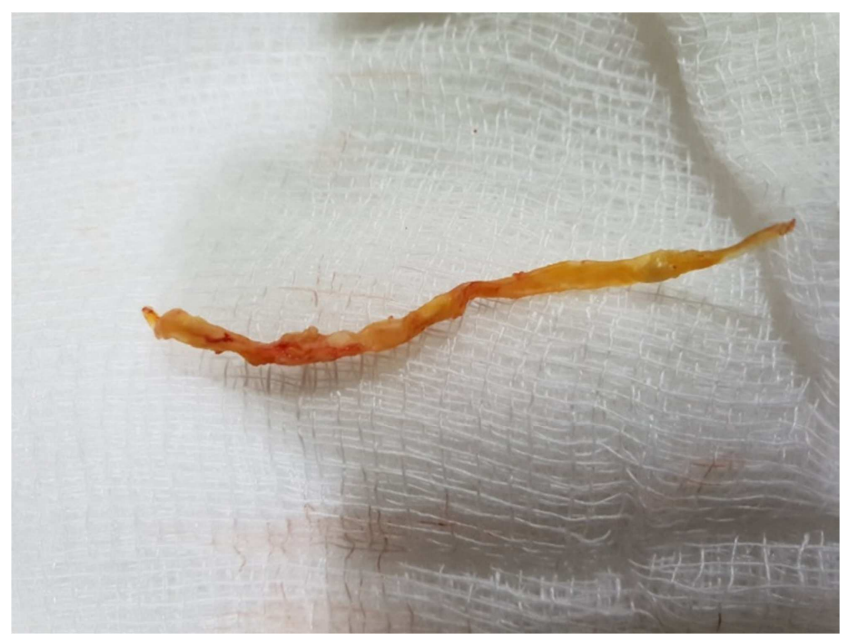

(a)

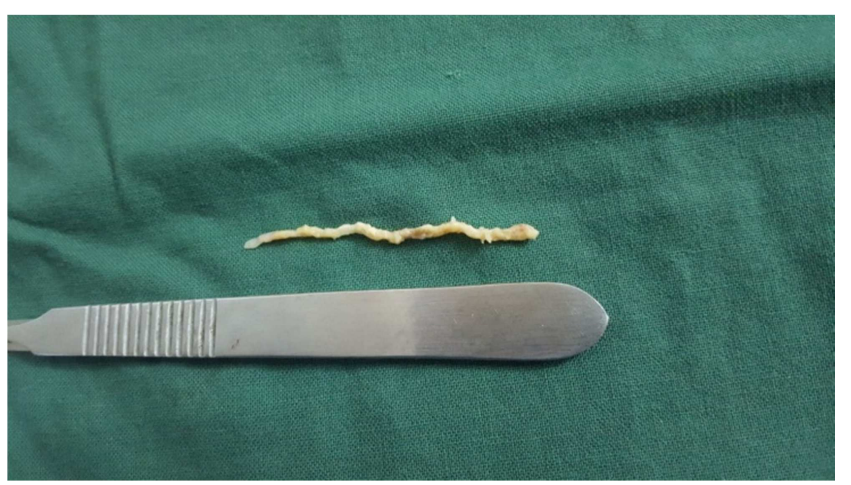

(b)

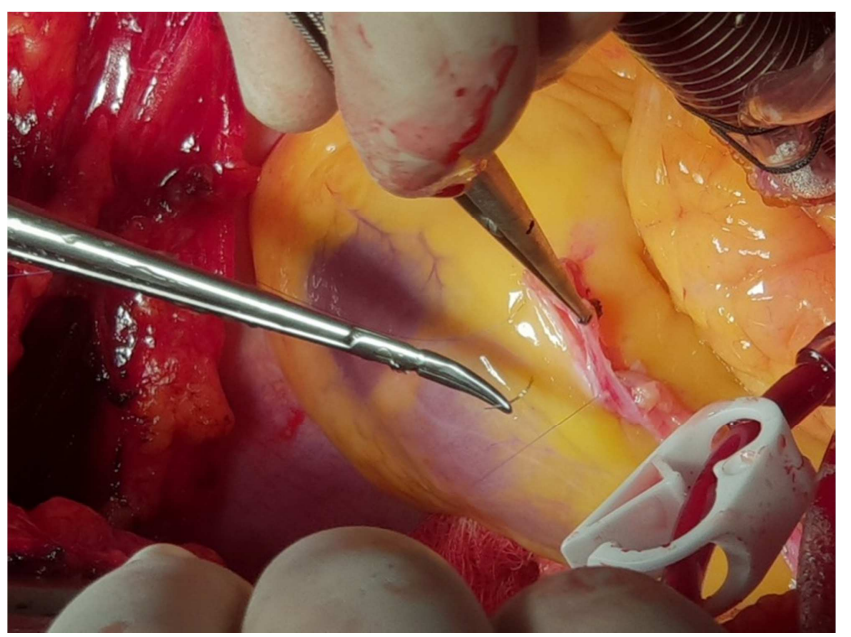

(c)

Figure 1. Intra operative photographs: (a) right coronary artery (RCA) endarterectomy specimen (b) left anterior descending coronary artery (LAD) specimen (c) left anterior descending coronary artery was reconstructed with long segmental patch plasty anastomosis with left internal mammary artery.

\section{Result}

Clinical and Echocardiographic Results 
A total of 22 patients underwent coronary endarterectomy with arterial and vein patch plasty of left anterior descending artery (LAD), Diagonal branch, right coronary artery (RCA) and posterior descending artery (PDA) with left internal mammary artery (LIMA) and saphenous vein graft (SVG). assessment done on outpatient department (OPD) basis clinically and hemodynamically by echocardiography, 7 patients had left ventricle ejection fraction of (50-55\%) preoperatively with no changes in postoperative period but 7 patients with ejection fraction of (30-35\%) preoperatively had improved to $(40-45 \%)$ in postoperative period with excellent clinical outcomes.

\section{Discussion}

A principal goal in coronary artery bypass grafting (CABG) surgery is to achieve complete revascularization of diseased coronary arteries, in particular the left anterior descending coronary artery (LAD) is a very important vessel because incomplete revascularization of the LAD has been proven to predictor of worse mortality after coronary artery bypass grafting surgery (CABG) [17]. However the diffusely diseased left anterior descending artery (LAD) and right coronary artery (RCA) remain a challenge for both percutaneous coronary intervention $(\mathrm{PCI})$ and coronary artery bypass grafting surgery (CABG), diffusely diseased coronary arteries have been treated by endarterectomy from the beginning of coronary artery surgery, recent publication have revealed that endarterectomy is a safe procedure and demonstrated favorable long term outcomes [15]. In our experience, endarterectomy has been performed with good clinical and echocardiographic outcomes. In the present study the mortality rate of patients undergoing endarterectomy was $0 \%$ which is not similar to other reports, the relatively higher mortality for endarterectomy compared with conventional coronary artery bypass grafting surgery $(\mathrm{CABG})$ may arise through associated comorbidities and risk factors rather than the use of endarterectomy in patients [10].

The major aim of endarterectomy is to establish sufficient blood supply to all branches left anterior descending artery (LAD) and diagonal branches and septal perforators, right coronary artery and posterior descending artery (RCA and PDA) with septal perforators that suffer from diffusely lying plaque, simple anastomosis to the distal part of these arteries cannot achieve sufficient blood supply to the important side branches, endarterectomy can eradicate the sequence of plaque and directly supply blood flow to the vessels, [11] another advantage of endarterectomy is the ability of obtain an anastomosis site when there are sever calcification of soft plaque that do not allow the performance of typical anastomosis. [2].

The optimal technique for performing endarterectomy remains controversial, there are many surgical methods, touch the plaque technique designated the closed and open technique, the closed technique is carried out by traction of the endarterectomized intima through a small arteriotomy. [1] Although it does not take much time, and anastomosis of the graft is easy, there are several possible disadvantages. These disadvantages include the possibilities that diagonal branches and septal perforators may be torn off despite gentle traction, and that the distal end of the lumen may become occluded with a thrombus or dissection owing to insufficient endarterectomy [6]. On the other hand, although the open method (long arteriotomy and total removal of plaque under direct visualization) requires a longer time, the openings of the side branches and distal end of the LAD and RCA can be directly observed and confidently endarterctomized using this method. Furthermore, we can fix the divided intima of the distal LAD and RCA to secure the distal flow using this method. We have adopted the open method with good results for LAD and closed method for RCA and PDA.

Recent investigation have shown that endarterectomy without use of cardiopulmonary bypass (off pump CABG) can be perform safely [12-14], but we consider that sever diffusely diseased coronary arteries should be perform using (on- pump CABG) method. [16]

The major cause for sub optimal result after coronary endarterectomy are related to triggering of the coagulation cascade by lake of endothelium in the early stage, tight management of antiplatelet and anticoagulant should be given after endarterectomy. [9] there was significant difference between the preoperative and postoperative left ventricle ejection fraction, we found local functional improvement of the anteroseptal myocardium [17].

\section{Conclusion}

Principle goal in coronary artery bypass grafting surgery (CABG) is to achieve complete revascularization of diseased coronary arteries, and it is not remain only for left anterior descending artery (LAD). arteries of lateral wall of left ventricle, obtus marginal (OM) branches of left circumflex artery and posterior descending artery (PDA) are very important vesseles as well. Recent publications have revealed that coronary endarterectomy is a safe procedure and demonstrated favorable long term out comes. In our experience coronary endarterectomy with good techniques, long arteriotomy with touch the plaq technique and arterial or vien patch plasty has been performed with good clinical and hemodynamic outcomes with echocardiography.

\section{References}

[1] Nishigawa K, Fukui T, Takanashi S. Coronary endarterectomy for the diffusely diseased coronary artery. Gen Thorac. Cardiovasc Surg 2014; 62: 461-7.

[2] Wang J, Gu C, Yu W, Gao M, Yu U. Short- and long-term patient outcomes from combined coronary endarterectomy and coronary artery bypass grafting: a meta-analysis of 63, 730 patients (PRISMA). Medicine (Baltimore) 2015; 94: e1781.

[3] Akgun S, Isbir CS, Yildirim T, Civelek A, Arsan S. Results of adjunctive coronary endarterectomy in 548 patients. Heart Surg Forum 2008; 11: E66-70. 
[4] Marinelli G, Chiappini B, Di Eusanio M, Di BartolomeoR, Caldarera I, Marrozzini C, MarzocchiA, Pierangeli A. Bypass grafting with coronary endarterectomy: immediate and longterm results. J Thorac Cardiovasc Surg 2002; 124: 553-60.

[5] Byrne JG, Karavas AN, Gudbjartson T, Leacche M, Rawn JD, Couper GS, Rizzo RJ, Cohn LH, Aranki SF. Left anterior descending coronary endarterectomy: early and late results in 196 consecutive patients. Ann Thorac Surg 2004; 78: 867-73.

[6] Fukui T, Takanashi S, Hosoda Y. Long segmental reconstruction of diffusely diseased left anterior descending coronary artery with left internal thoracic artery with or without endarterectomy. Ann Thorac Surg 2005; 80: 2098-105.

[7] Myers PO, Tabata M, Shekar PS, Couper GS, Khalpey ZI, Aranki SF. Extensive endarterectomy and reconstruction of the left anterior descending artery: early and late outcomes. J Thorac Cardiovasc Surg 2012; 143: 1336-40.

[8] Sirivella S, Gielchinsky I, Parsonnet V. Results of coronary artery endarterectomy and coronary artery bypass grafting for diffuse coronary artery disease. Ann Thorac Surg 2005; 80: 1738-44.

[9] Schwann TA, Zacharias A, Riordan CJ, Durham SJ, Shah AS, Habib RH. Survival and graft patency after coronary artery bypass grafting with coronary endarterectomy: role of arterial versus vein conduits. Ann Thorac Surg 2007; 84: 25-31.

[10] Tiruvoipati R, Loubani M, Peek G. Coronary endarterectomy in the current era. Curr Opin Cardiol 2005; 20: 517-20.

[11] Shapira OM, Akopian G, Hussain A, Adelstein M, Lazar HL,
Aldea GS, Shemin RJ. Improved clinical outcomes in patients undergoing coronary artery bypass grafting with coronary endarterectomy. Ann Thorac Surg 1999; 68: 2273-8.

[12] Eryilmaz S, Inan MB, Eren NT, Yazicioglu L, Corapcioglu T, Akalin H. Coronary endarterectomy with off-pump coronary artery bypass surgery. Ann Thorac Surg. 2003; 75: 865-9.

[13] Vohra HA, Kanwar R, Khan T, Dimitri WR. Early and late outcome after off-pump coronary artery bypass graft surgery with coronary endarterectomy: a single-center 10-year experience. Ann Thorac Surg 2006; 81: 1691-6.

[14] Fukui T, Takanashi S, Hosoda Y, Suehiro S. Early and midterm results of off-pump coronary artery bypass grafting. Ann Thorac Surg 2007; 83: 115-9.

[15] Kleisli T, Cheng W, Jacobs MJ, Mirocha J, Derobertis MA, Kass RM, Blanche C, Fontana GP, Raissi SS, Magliato KE, Trento A. In the current era, complete revascularization improves survival after coronary artery bypass surgery. J Thorac Cardiovasc Surg 2005; 129: 1283-91.

[16] Nathoe HM, Buskens E, Jansen EW, Suyker WJ, Stella PR, Lahpor JR, van Boven WJ, van Dijk D, Diephuis JC, Borst C, Moons KG, Grobbee DE, de Jaegere PP. Role of coronary collaterals in off-pump and on-pump coronary bypass surgery. Circulation 2004; 110: 1738-42.

[17] Shapira OM, Akopian G, Hussain A, et al. Improved clinical outcomes in patients undergoing coronary artery bypass grafting with coronary endarterectomy. Ann Thorac Surg 1999; 68: 2273-8 